\title{
Experience of Tympanoplsty Done Under Local Anesthesia without Sedation
}

\author{
${ }^{1}$ Md. Ashraful Islam MBBS, FCPS, FICS, FRCS (Glasgow), Professor and Head, Otolaryngology-Head \& Neck Surgery, \\ Bangladesh Medical College Hospital \\ ${ }^{2}$ Tareq Mohammad MBBS, FCPS, Assistant Registrar ENT, Bangladesh Medical College Hospital \\ ${ }^{3}$ Nazmul Hossain Chowdhury, MBBS, FCPS, Assistant Professor, ENT, Bangladesh Medical College Hospital \\ ${ }^{4}$ Towsif Bin Mamoon, MBBS, Assistant Registrar, ENT, Bangladesh Medical College Hospital \\ ${ }^{5}$ Farid Uddin Milki, MBBS, DLO Consultant ENT, Bangladesh Medical College Hospita \\ ${ }^{6}$ ASM Lutfur Rahman MBBS, MCPS, DLO, MRCPS (Glasgow), Resident Surgeon, Bangladesh Medical College Hospital \\ ${ }^{7}$ Saif Rahman Khan MBBS, Assistant registrar, ENT, National Institute of ENT
}

\section{Abstract}

\section{$>$ Background \& Objectives:}

Tympanoplsty is the common operation for chronic otitis media that is performed under local Anesthesia (LA) or general anesthesia (GA). LA is more popular than $G A$ in the underdeveloped and developing countries in almost all ear surgeries. The authors have been using $L A$ without sedation for more than 20 years. LA without sedation is relatively safer, less expensive and perform more surgeries in a shorter period of time. This study aimed to share the experience of $L A$ without sedation in tympanoplsty.

\section{$>$ Patients and Method:}

7050 patents underwent tympanoplsty under LA without sedation from 1999 to 2019 in the different hospital of Bangladesh. Patients were thoroughly evaluated preoperatively, explained the advantages of LA without sedation in details $L A$ was used with different Adrenaline concentrations (1: 1000 to 1: 200,000) either by injection or topically. Patients' compliance, pain, discomfort, drowsiness, and other parameters ware recorded.

\section{$>$ Results:}

Amongst 7050 patients, 3877 were male and 3173 were female. The youngest patient was 14 year boy and oldest patient was of 58 year male. Operative time was short with a bloodless field. Mean operative time was 45 minutes. The overall safety was good and needed shortest hospital stay (Hour/day); and of course very less expensive.

\section{Conclusion:}

Tympanoplsty under local anesthesia without sedation is safer, relatively cost effective, can be performed as a day/hour care case. Suitable patient selection and adequate preoperative counseling is essential to achieve a great success.

Keywords:- LA, no sedation, tympanoplsty, safe, less expensive.

\section{INTRODUCTION}

Tympanoplasty is one of the common procedures that an Otolaryngologist does in their regular practice. Most of the ENT surgeons are doing this surgery under general anesthesia since long but local anesthesia is becoming popular in the underdeveloped and developing countries. Local anesthesia with preoperative sedation are being used to the patient to have a tolerable patients' surgeons' comfort. In this study authors have been doing tympanoplsty under local anesthesia without using sedation for the last 20 years. The patient is discharged on the same day or even an hour after the operation so it can be termed as a day or even an hour care surgery.

Surgery under local anesthesia has several advantages with fewer complications though at times life threatening complications may happen. But when sedative or premedication is added to local anesthetic agents, a large group of surgeons experience some common hazards like sudden aerosol, pain intolerance, respiratory depression, lack of co-operation; and inadequate sedation which requires frequent intraoperative dose adjustment. Presence of an anesthetist is also important during surgery with sedation, though may not be available every time. Cost of surgery is an important factor in the underdeveloped and developing country. Because of the financial burden many of the patients do not turn up for this surgery until and unless it deem essential for treatment. Presence of an anesthetist with a monitor for continuous monitoring of patients cardiac status and overall conditions doubles the cost of surgery indeed. Although LA can be used in a wide range of otologic surgeries, including myringoplasty, tympanoplsty, myringotomy,ossiculoplasty, mastoidectomy, facial nerve decompression and stapes surgery ${ }^{1}$, only a limited number of otolaryngologists adopt it $(20 \% \text { in UK) })^{2,3}$

Considering the aforementioned scenario, the authors have been doing tympanoplsty under local anesthesia without sedation for more than 20 years and experienced no significant intraoperative or postopetive problem. In this retrospective study we included 7050 patients who underwent tympanoplsty under local anesthesia without any type of preoperative or intraoperative sedation. 


\section{$>$ Objectives}

- To share the experience of using LA without sedation in tympanoplsty

- To find out patients compliance during the operative procedures without sedation.

\section{PATIENTS AND METHODS}

Study design: Retrospective study

Study sample: 7050 patients with indication for tympanoplsty.

Sampling technique: Simple non-random sampling

Study period: 20 years (1999-2019)

Study places:

- Bangladesh Medical College Hospital

- Popular Medical College Hospital

- Other district hospitals of Bangladesh

\section{$>$ Inclusion criteria}

- Age: 10 year to 60 year.

- Tympanic membrane perforation

\section{$>$ Exclusion criteria}

- Patients unfit for surgeries

- Chronic squamous otitis media

- Patients not giving consent.

\section{$>$ Procedure}

The most important aspect of this procedure is preoperative counseling and motivation which is done in the otolaryngologist's outpatient clinic. Almost all patients became concerned about neck pain assuming prolonged operative time experienced in their previous surgical procedures, type of anesthesia to be used and their comorbidities. During this counselling session we tried our best to explain their above queries, earned their confident, became motivated; and finally obtained informed consent of the procedure. Operative steps ware described, assured regarding neck movement time to time if required and also they were taught how to cooperate during surgery. It is a day/hour care procedure, so every patient would be discharged on same day. We had noticed awareness regarding cost of surgeries; but at the same time expected a good surgery with minimum cost. So it had drawn an important impact during counseling time. To reduce the anxiety of surgery almost every patients were advised to take 1 anxiolytic drug at night before surgery.

During surgery, surgical field prepared in the standard fashion with povidone-iodine and head positioned on a head ring. The draping were applied in such a way that the patient could able to watch his surgery on the monitor if he or she desired.

The author used prepared anesthetic solution (AS) with $2 \%$ lidocaine solution with $1: 1000$ to a concentration of $1: 5000$ to $1: 100,000$ which was given by injection or topically. The concentration and dose based on the age and pre-existing cardiac disease (The total amount of injected
AS should not exceed $7 \mathrm{mg} / \mathrm{kg}$ body weight, $20 \mathrm{ml}$ in average adult). In most patients, 12 to $15 \mathrm{ml}$ AS was needed. Injection is strictly extravascular; the syringe piston is usually withdrawn before each injection to assure extravascular injection.

The preparation and administration of the solutions were done by the surgeon himself. Prior to infiltration, the patient was told that he or she might get a temporary discomfort. Four quadrant block was given to the external auditory canal

LA injection technique involved four quadrant points in the external auditory canal by using 26 gauge needle, 02 $\mathrm{ml}$ of each AS was injected in the canal in the 4 points of the external auditory canal namely 3, 6, 9 and 12 O' clock position.

- The bevel of the needle is directed toward the bone, and the AS was injected subperiosteally to avoid blebs or damage to the skin. The author modified the injection of bone meatus by introducing the needle in the thick skin of cartilagenous meatus, and then proceeding subcutaneously until it reaches the bone meatus to infiltrate it. This simple method helps to keep the integrity of the thin skin of the bone canal.

- The ME mucosa was anesthetized by placing the gel foam/small cotton ball soaked with AS through the tympanic membrane (TM) perforation. But in intact $\mathrm{TM}, 1 \mathrm{ml}$ of AS was instilled in the ME cavity once opened after elevation of the annulus and left for 1 $2 \mathrm{~min}$ to anesthetize the tympanic plexus.

- In the early postoperative period, patients were asked

- to score their discomfort for pain, noise, anxiety, irrita-

- bility (uneasiness), and position of the body and neck by

- means of a questionnaire. The scores ranged from " 0 " to

- " $4 \leqslant$ " for each question, " 0 " for no discomfort and " $4 \leqslant$ "

- for extreme discomfort.

- In the intraoperative \& early postoperative period, patients were asked to score their discomfort for pain, noise, anxiety, irritability (uneasiness) and position of the body and neck by a means of questionnaire. The scores ranged from " 0 " to " 4 " for each question, " 0 " for no discomfort and " 4 " for extreme discomfort.

- Additional questions were asked about the most disturbing sensation during the operation and whether they would prefer local anesthesia for a second procedure.

- Per operative bleeding \& difficulties faced by the surgeon were also noted. Operating time was also recorded. 


\section{RESULTS}

In our series, total 7050 patients were included, among them 3877 (55\%) were male and 3173 (45\%) were female. The youngest patient was 14 year boy and oldest patient was of 58 year male. In our series, we noticed that male patients consented easily for surgery under local anesthesia.

It was observed that those patients who underwent previous surgical procedures, they easily accepted this procedure under local anesthesia without sedation. As such among 7050 patients, $433(6.14 \%)$ patients had H/O surgery under local anesthesia, 231 (3.28\%) female patients had H/O surgery under spinal anesthesia, 121 (1.72\%) patients had $\mathrm{H} / \mathrm{O}$ surgery under $\mathrm{G} / \mathrm{A}$.

During surgery, we graded patients discomfort from 0 (no discomfort) to 4 (extreme discomfort) table 1. Most patients had some degree of neck movement; next problem was pain during initial injection, few complained procedural pain during flap elevation from attic region.

There was no significant per-operative complication except bleeding. Transient facial weakness occurred in 2 cases that was recovered few hours after surgery, might be due to injection along the tympano-mastoid suture line. All surgery done through end-aural approach. 5088 (72.17\%) cases done under microscope and rest 1962 (27.83\%) cases by endoscope, as the author initially accustomed with microscope.

Timing of surgery was variable ranging between 30 minutes to 1 hour, mean 45 minutes. All 5800 (82.29\%) patients of private chamber were discharged on same day and rest $1962(27.83 \%)$ on 1st POD especially those operated in Medical College.

\begin{tabular}{|c|c|c|c|c|c|}
\hline & \multicolumn{5}{|c|}{ Grade } \\
\hline & 0 & 1 & 2 & 3 & 4 \\
\hline Position of body and neck & $\begin{array}{c}1851 \\
(26.26 \%)\end{array}$ & $4850(64.96 \%)$ & $349(4.95 \%)$ & \\
\hline Pain & $\begin{array}{c}889 \\
(12.61 \%)\end{array}$ & $6055(85.89 \%)$ & $97(1.38 \%)$ & $9(0.12 \%)$ & \\
\hline Noise & $\begin{array}{c}3224 \\
(45.73 \%)\end{array}$ & $3500(49.65 \%)$ & $326(4.62 \%)$ & & $6(0.08 \%)$ \\
\hline Anxiety & $\begin{array}{c}5131 \\
(72.78 \%)\end{array}$ & $1851(26.26 \%)$ & $62(0.87 \%)$ & 6 \\
\hline
\end{tabular}

$\mathrm{O}=$ no discomfort, $1=$ mild discomfort, $2=$ moderate discomfort, $3=$ severe discomfort, $4=$ extreme discomfort.

Table 1:- scores for pain, body and neck position, noise, anxiety

\section{DISCUSSION}

Tympanoplasty under local anesthesia can be done comfortably and safely though most of the otolaryngologists do it under general anesthesia. Local and General anesthesia (GA) are two different entities. There are several established advantages of using local anesthesia includes less bleeding, less operative time, cost effectiveness, avoidance of hazards of general anesthetic drugs. Most important of these is per-operative facial nerve testing and hearing assessment.

Preoperative counseling is very important for any kind of surgery under local anesthesia. Patient's anxiety was more in those, who were not properly counseled. Pain sensation is variable among person to person depending on psychological status and previous experiences. Sometimes patient experiences much pain than that of surgical trauma. Patient selection is also important.

There are several studies of Tympanoplasty under local anesthesia with sedation. Raghu S. Thuta ${ }^{4}$ and B Edussuriya $^{5}$ showed some problem they faced during surgery like inappropriate movement, nausea vomiting, snoring, and hypotension. There is an established known risk of sedative like respiratory depression. We didn't experience any such problem in our series as our works done without sedation.

Significant adverse effects of LA did not found in many studies on local anesthesia for middle ear surgery ${ }^{6,7}$. Excellent analgesia can be achieved by blocking the branch of great auricular nerve (retroauricular infiltration) and tympanic branch of auriculotemporal nerve ( $\mathrm{V}$ shaped infiltration). We also observed no significant complication during our surgery.

In our series total 7050 patients were operated under LA without sedation. Among them 3877 (55\%) were male and $3173(45 \%)$ were female. The eldest patient was 14 year and oldest patient was of 58 years. We observed that male patients easily accepts the procedure.

There was another observation that, those have previous history of surgery, easily accepts the procedure. In our series, $433(6.14 \%)$ patients had $\mathrm{H} / \mathrm{O}$ surgery under local anesthesia, 231 (3.28\%) female patients had $\mathrm{H} / \mathrm{O}$ surgery under spinal anesthesia, $121(1.72 \%)$ patients had $\mathrm{H} / \mathrm{O}$ surgery under $\mathrm{G} / \mathrm{A}$. It was easier to counsel them for surgery under local anesthesia and they were well cooperated during the procedure. 
It has been reported that, sensation of noise and anxiety were the most common discomforts but the majority of patients preferred local anesthesia ${ }^{8,9}$.In our questionnaire survey, patients scored their discomfort of pain, body and neck movement, anxiety and noisy sensation from grade 0 (no discomfort) to 4 (extreme discomfort), corresponding to a value lower than " 1 " indicating "mild." Most patients 6055 (85.89\%) experienced pain only at the beginning of the surgery because of numerous injections of the local anesthetic. Pain score was grade 1 among these $85.89 \%$ patients. 97 $(1.38 \%)$ patients had grade 2 pain score and only $9(0.12 \%)$ had grade 3 pain score. Almost every patient was informed that they would be allowed to move their body and neck up to certain range during operation. Although 4850 (64.96\%) patients had grade 1 discomfort regarding movement. 349 $(4.95 \%)$ patients had grade 2 and none of them had grade 3 or $4.3500(49.6 \%)$ patient had grade 1 noise discomfort, especially due to instrumentation. $326(4.62 \%)$ had grade 2 and none on grade 3 or 4 . Maximum patients, \{5131 $(72.78 \%)\}$ was not anxious or complained of fear of surgical procedure during surgery though 1851 (26.6\%) patients were anxious about the procedure and outcome of surgery at the time of procedure and categorized as grade 1 , $62(0.87 \%)$ was of grade 2 , and $6(0.08 \%)$ patients had grade 3 discomfort and needed further counseling. Our experience corresponds to previously mentioned studies.

In our series, hearing was assessed during the operations using free-field audiometry. In a successful hearing reconstruction, the patient heard whispered voice at $1 \mathrm{~m}$ distance. Here we followed Mohamed A et $\mathrm{al}^{18}$.

Per operative facial nerve testing by asking the patient to smile and closing the eye is a good alternative to facial nerve monitor. However, sometimes, temporary FN paralysis may occur due to excessive injection at the mastoid tip or on the lateral surface of the tragus, which may be explained by trickling of AS along the tragus to the tragal pointer. We observed 2 cases of transient facial palsy at the end of type 1 tympanoplasty that recovered within few hours after surgery. It may be happened due to trikling of LA solution along the tympanomastoid suture line to the facial nerve. Lancer and Fisch ${ }^{4}$ had a higher incidence of $\mathrm{FN}$ anesthesia due to injection in the region of the stylomastoid foramen. Using their injection technique in a series of 32 patients, they reported that $97 \%$ developed a temporary postoperative facial weakness. In our study we operated maximum patients under microscope with endaural approach, endoscope also used in many case. We did not used postauricular route, so FN deficit may be less in number in our study.

Regarding safety, it is safer to use LA rather GA, mentioned by different authors in their literature. The established complications of GA are following: risk for laryngotracheal trauma by means of endotracheal intubation, cardiopulmonary and hepatic complications due to GA drugs. But in case of local anesthesia we can easily avoid these. There is less postoperative nausea and vomiting, and, in general, mobilization is early ${ }^{10,11}$.
We used Lidocaine as local anesthetic and combined with adrenaline in different concentration to prolong its action depending upon patient's age and cardiac status. It is characterized by a rapid onset and prolong duration of action ${ }^{12,13}$.Due to the vasoconstrictive action of adrenaline it significantly reduces the bleeding, hence allows less operative time as well. Because of its action causing hypotension, bradycardia, and even cardiac arrest, lidocaine use is contraindicated in patients with second or third degree heart block (without pacemaker), severe sinoatrial block (without pacemaker), hypotension, and bradycardia ${ }^{14-}$ ${ }^{16}$. Systemic exposure to excessive quantities of lidocaine mainly results in central nervous system and cardiovascular effects. So, careful purely extravascular infiltration is must.

Addition of adrenaline prolongs its action and decreases its toxicity. It is recommended not to infiltrate lidocaine more than $4.5 \mathrm{mg} / \mathrm{kg}$ without adrenaline, but with adrenaline it can reach $7 \mathrm{mg} / \mathrm{kg}$. Pure extravascular injection is must to avoid toxicities and rubbing the skin after injection is also prohibited in some studies, though in our series in almost every case we rubbed after infiltration. In our series, it was found that the time of action of lidocaine was nearly doubled if adrenaline 1:200000 was added and increased by 2 fold again with 1: 20000 adrenaline. This is in agreement with the opinion of Neal et al. ${ }^{17}$, who stated that adrenaline vasoconstricts arteries, reducing bleeding, and delays the resorption of lidocaine, almost doubling the duration of anesthesia.

Throughout the study we noted that, success of the technique depends on following factors: expertise of the surgeon, careful monitoring, and acceptance of some movement during surgery and obviously suitable patient selection. We found in our series that very young and very anxious patients are most unsuitable candidate for surgery under local anesthesia.

Because of the low cost, early recovery, and shorter hospital stay (day cases), LA was used successfully by the author in the ear surgery in different tertiary level hospitals.

\section{CONCLUSION}

Tympanoplsty under local anesthesia without sedation is safer, relatively cost effective, can be performed as a day/hour care case. The advantages are innumerable in terms of patients' comfort, less bleeding, ability to assess hearing during surgery and of course cost effective. Suitable patient selection and adequate preoperative counseling is essential to achieve a great success.

\section{REFERENCES}

[1]. Caner G, Olgun L, Gültekin G, Aydar L. Local anesthesia for middle ear surgery. Otolaryngol Head Neck Surg 2005;133(2):295-297.

[2]. Hollinshead WH. Anatomy for surgeons: Vol 1. The head and neck. Philadelphia: Lippincott Williams \& Wilkins; 1982 
[3]. Peuker ET, Filler TJ. The nerve supply of the human auricle. ClinAnat 2002; 15:35-7

[4]. Lancer JM, Fisch U. Local anesthesia for middle ear surgery. ClinOtolaryngol 1988;13:367-74.

[5]. Andreassen UK, Larsen CB. [Anesthesia in ear surgery. A source economical analysis and patient assessment of general anesthesia versus local anesthesia in ear surgery]. UgeskrLaeger 1990;28;152:1595-7.

[6]. Abdul-Latif RM. Stapedectomy data base study [MS degree in OLR]. Cairo, Egypt: Faculty of Medicine, Ain Shams University; 2012

[7]. Martha R. Amoroch c. Anesthesia for Tympanomastoidectomy.Anesthesiology pp 281285

[8]. Sarmento KM Jr, Tomita S. Retroauriculartympanoplasty and tympanomastoidectomy under local anesthesia and sedation. ActaOtolaryngol 2009;129(7):726-728

[9]. Pöntinen PJ. Cardiovascular effects of local adrenaline infiltration during halothane anaesthesia and adrenergic beta-receptor blockade in man. ActaAnaesthesiolScand 1978;22(2):130-144

[10]. Becker DE, Reed KL. Essentials of local anesthetic pharmacology. AnesthProg 2006;53(3):98-108. quiz 109-110.

[11]. Jackson D, Chen AH, Bennett CR. Identifying true lidocaine allergy. J Am Dent Assoc 1994;125(10):1362-1366.

[12]. Abdellatif AA, Elkabarity RH, Hamdy TA. Dexmedetomedinevs midazolam sedation in middle ear surgery under local anesthesia: effect on surgical field and patient satisfaction. Egypt $\mathrm{J}$ Anaesth 2012;28:117-123.

[13]. GhanemAM.Cartilagetympanoplasty(type1)bylocalan aesthesia 'Costs and Results' Thesis Submitted for partial Fulfillment of MS degree in ORL. Cairo, Egypt: Al Azhar University; 2003.

[14]. Nader ND, Simpson G, Reedy RL. Middle ear pressure changes after nitrous oxide anesthesia and its effect on postoperative nausea and vomiting. Laryngoscope. 2004; 114(5):883-6.

[15]. Neal JM, Bernards CM, Butterworth JF 4th, di Gregorio G, Drasner K, HejtmanekMRet al. ASRA practice advisory on local anesthetic systemic toxicity. RegAnesth Pain Med 2010;35(2):152-161. 